\title{
Genetic structure of community acquired methicillin-resistant Staphylococcus aureus USA300
}

Ryan Tewhey ${ }^{1,2,3 \dagger}$, Christopher R Cannavino ${ }^{4,5,6 \dagger}$, John AD Leake ${ }^{4,5}$, Vikas Bansal ${ }^{1}$, Eric J Topol ${ }^{1,2}$, Ali Torkamani ${ }^{1,2}$, John S Bradley ${ }^{4,5^{*}}$ and Nicholas J Schork ${ }^{1,2^{*}}$

\begin{abstract}
Background: Community-associated methicillin-resistant Staphylococcus aureus (CA-MRSA) is a significant bacterial pathogen that poses considerable clinical and public health challenges. The majority of the CA-MRSA disease burden consists of skin and soft tissue infections (SSTI) not associated with significant morbidity; however, CA-MRSA also causes severe, invasive infections resulting in significant morbidity and mortality. The broad range of disease severity may be influenced by bacterial genetic variation.

Results: We sequenced the complete genomes of 36 CA-MRSA clinical isolates from the predominant North American community acquired clonal type USA300 (18 SSTI and 18 severe infection-associated isolates). While all 36 isolates shared remarkable genetic similarity, we found greater overall time-dependent sequence diversity among SSTI isolates. In addition, pathway analysis of non-synonymous variations revealed increased sequence diversity in the putative virulence genes of SSTI isolates.

Conclusions: Here we report the first whole genome survey of diverse clinical isolates of the USA300 lineage and describe the evolution of the pathogen over time within a defined geographic area. The results demonstrate the close relatedness of clinically independent CA-MRSA isolates, which carry implications for understanding CA-MRSA epidemiology and combating its spread.
\end{abstract}

\section{Background}

For the past 75 years, successive waves of antibioticresistant Staphylococcus aureus strains have posed significant challenges to clinicians and public health officials [1]. Beta-lactamase-mediated $S$. aureus resistance developed within a decade of widespread penicillin use, and two years after the introduction of methicillin, methicillinresistant $S$. aureus strains (MRSA) appeared. More recently, MRSA has emerged as one of the preeminent public health threats in the United States and worldwide. In fact, all-cause $S$. aureus infections have rapidly increased during the past decade (1999, 294,570 annual U.S. cases vs. $2005,477,927$ cases) with MRSA currently accounting for $>50 \%$ of staphylococcal disease $[2,3]$. More deaths were attributed in 2005 in the U.S. to

\footnotetext{
* Correspondence: jbradley@rchsd.org; nschork@scripps.edu

${ }^{\dagger}$ Equal contributors

${ }^{4}$ Department of Pediatrics, Division of Infectious Diseases, Rady Children's

Hospital San Diego, San Diego, CA, USA

${ }^{5}$ Department of Pediatrics, Division of Infectious Diseases, University of California, San Diego, CA, USA

Full list of author information is available at the end of the article
}

S. aureus than HIV/AIDS [3]. MRSA also represents a significant economic burden with an estimated annual U.S. cost of 9 billion dollars [2]. Historically the MRSA threat consisted of hospital-acquired strains (HA-MRSA) usually affecting individuals with associated risk factors (e.g., hospitalization, multiple antibiotics).

Community-associated MRSA strains (CA-MRSA) have recently emerged as the predominant cause of MRSA disease [4]. Distinct genetic profiles suggest that CA-MRSA and HA-MRSA evolved independently. While methicillin-resistance occurs at a fitness cost to the organism in HA-MRSA strains, CA-MRSA strains have a selective advantage and generally affect previously healthy individuals [5,6]. CA-MRSA strains carry unique drug resistance genes, and widespread clinical experience suggests they may possess increased virulence compared to most HA-MRSA.

While recombination is rare among staphylococci, broad diversity exists amongst subspecies. Among CA-MRSA strains, one clonal isolate, USA300, has become predominant in the United States, representing the majority

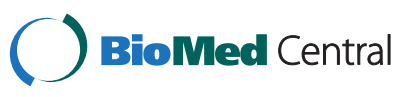


of all MRSA infections and almost all communityassociated staphylococcal infection in much of the U.S. $[3,4,7]$. Its prevalence is also increasing in Europe [8,9]. USA300's broad range of clinical manifestations include asymptomatic colonization, skin and soft tissue infections (SSTI), and life-threatening, severe disease (e.g. complicated pneumonia, endocarditis, osteomyelitis, and other organ-specific pathology) [10]. Given this clinical diversity, there may exist genetic differences that could serve as useful predictors of CA-MRSA-related disease phenotypes or virulence. Classical genotyping methodologies such as PFGE and MLST rely on the evaluation of highly conserved housekeeping genes representative of the vertical gene pool and resultantly provide insufficient resolution to predict disease phenotypes. Recent reports suggest that there are additional genetic components that contribute to invasiveness not captured by current typing technologies [11].

In an attempt to understand the population genetic architecture of CA-MRSA isolates and possibly resolve genomic differences relating to MRSA invasiveness, we sequenced the whole genomes of 36 CA-MRSA clinical isolates. By leveraging novel de novo assembly methods along with the historical time-stamps associated with the infections, we sought to determine whether there was evidence for CA-MRSA selection both across all coding genes as well as biological pathways implicated to mediate virulence.

\section{Results}

\section{Clinical collection}

During 2001-2006, 925 children with MRSA infections were identified at Rady Children's Hospital in San Diego, California. To prevent the inclusion of hospital acquired strains we excluded 413 isolates (44.6\%) (non-sterile sites, polymicrobial infections, chronic disease requiring frequent hospitalization, etc.). Among 512 remaining children, 41 (8\%) had severe infections (among whom 36 had viable frozen isolates) and 471 (92\%) had SSTI. 36 CA-MRSA SSTI-associated isolates of the same chronological distribution as that of the severe isolates were selected for genotypic comparison. The majority of the clinical diagnoses of the 36 severe CA-MRSA infections consisted of either osteoarticular infections (52.8\%) or complicated pneumonias (30.6\%).

There was no significant difference in patient age, gender or self-identified ethnicity between the severe infection and SSTI study groups. Compared with children with SSTI, children with severe infections had significantly longer hospital stays, longer duration of intravenous antibiotic therapy, and total duration of antibiotics (Additional file 1: Table S1).

Of all children with CA-MRSA severe infections, 3 (8.3\%) children died, another 5 (13.9\%) required intensive care prior to recovery, and 10 others $(27.8 \%)$ were hospitalized for $\geq 10$ days prior to recovery. Further, two children (5.6\%), both with osteomyelitis, had longterm sequelae (chronic osteomyelitis and distal radius growth arrest as documented on follow-up) (Additional file 1: Table S2 and Additional file 1: Table S3). All children in the CA-MRSA SSTI group were hospitalized $<10$ days and had an uncomplicated recovery during hospitalization.

Multilocus PCR coupled to electrospray ionization-mass spectrometry (PCR/ESI-MS) genotyping revealed that all 72 severe infection and SSTI-associated CA-MRSA isolates were of a single genotype: PCR/ESI-MS genotype 1, which corresponds to PFGE USA300/USA500 and sequence type 8 designations [12]. We selected 36 isolates representing the entire collection period for whole genome sequencing (severe infections $\mathrm{n}=18$; SSTI $\mathrm{n}=18$ ). In addition, two replicate DNA samples from the USA300 strain FPR3757 (which has a high quality complete genome in the public database) were sequenced to serve dual functions of quality control and assistance during sequence assembly [7].

\section{Sequencing and data summary}

We sequenced CA-MRSA isolates using the Illumina Genome Analyzer II. In order to maximize sequencing capacity, we sequenced 3 or 4 isolates per lane using nucleotide barcodes, with an average of $181 \mathrm{Mb}$ of sequence per isolate. We used a custom pipeline that leveraged remapping protocols using the aligner BWA [13] against the closely related reference sequence USA300-FPR3757 as well as de novo assembly with Abyss [14] to successfully call polymorphisms ranging from single nucleotide variants (SNV) to large structural rearrangements. Of the core genome defined as the curated FPR3757 sequence, we sequenced to an average depth of 57 -fold with $99.9 \%$ of bases across all genomes covered $>5 \mathrm{x}$ and $93.8 \%>20 \mathrm{X}$ (Table 1).

In order to prevent false positive calls, we conservatively masked $3 \%$ of the genome where placing or assembling short reads could not be confidently performed. All single nucleotide events as well as large insertions and deletions that originated in the masked region were discarded. We also detected the presence of all four previously identified USA300 plasmids in our isolates. The most common combination was the presence of pUSA01 and pUSAHOU300 in 22 of the isolates (Figure 1). The remaining 14 strains represented an additional 4 combinations that is not delineated by the phylogenetic structure of the population. As noted, in addition to the 36 clinical CA-MRSA isolates, we sequenced the FPR3757 reference strain in duplicate. Perfect concordance was obtained between the duplicates with each sample having 24 detected variants compared to the 


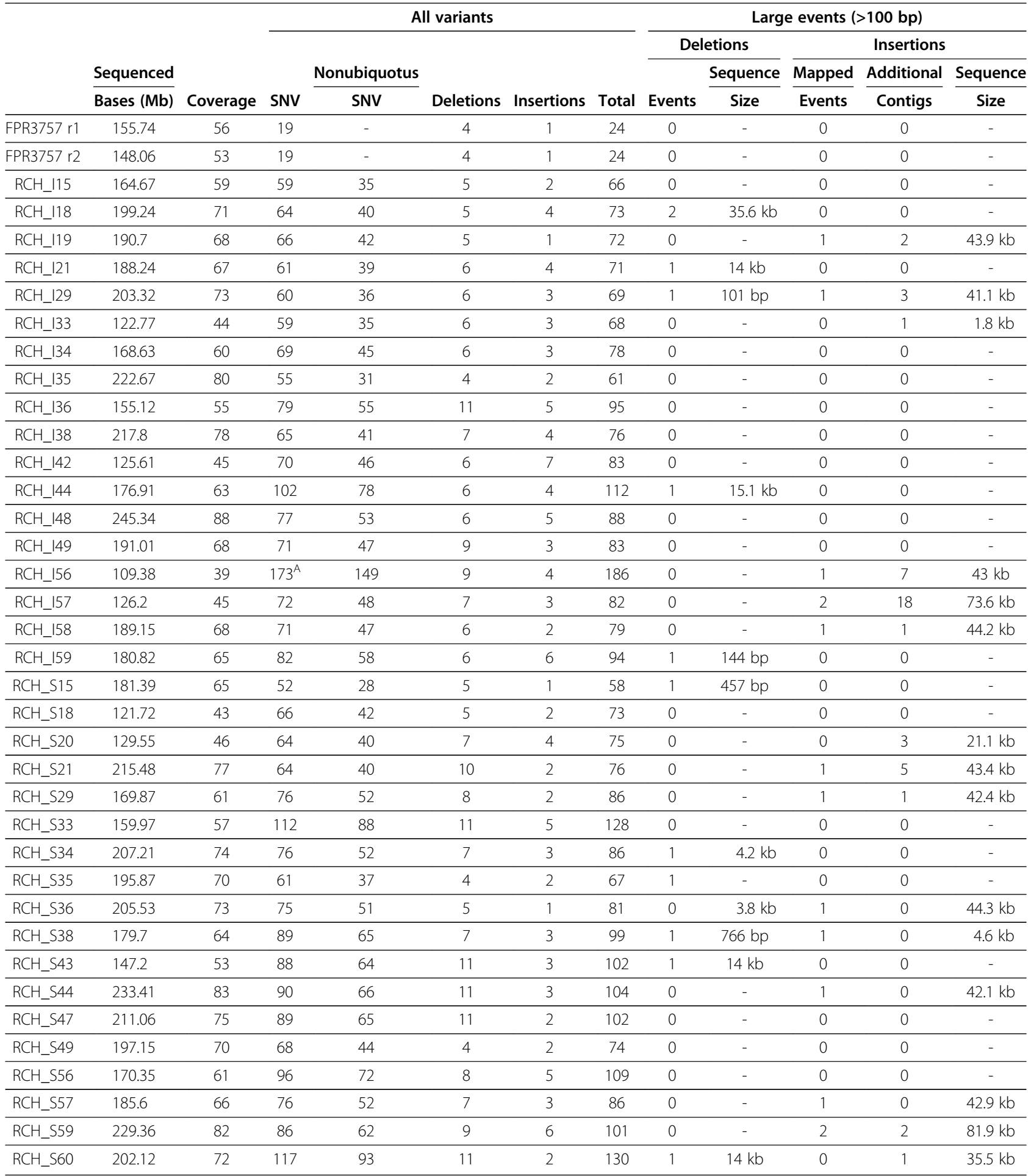

A - 84 SNV \& 1 insertion confined to a single $10 \mathrm{~kb}$ stretch were removed from all subsequent analysis.

reference sequence. Of the 24 variants, 17 were previously reported as probable errors in the reference sequence [15]. We identified 7 new variants in FPR3757, 5 of which were seen in all clinical isolates, supporting their status of true variants. The remaining two variants were visually inspected and determined as true variants. We also detected one large rearrangement in the reference sequence consisting of a $13.3 \mathrm{~kb}$ relocation. This rearrangement had been previously reported in a separate USA300 strain [15] and was also detected in all 


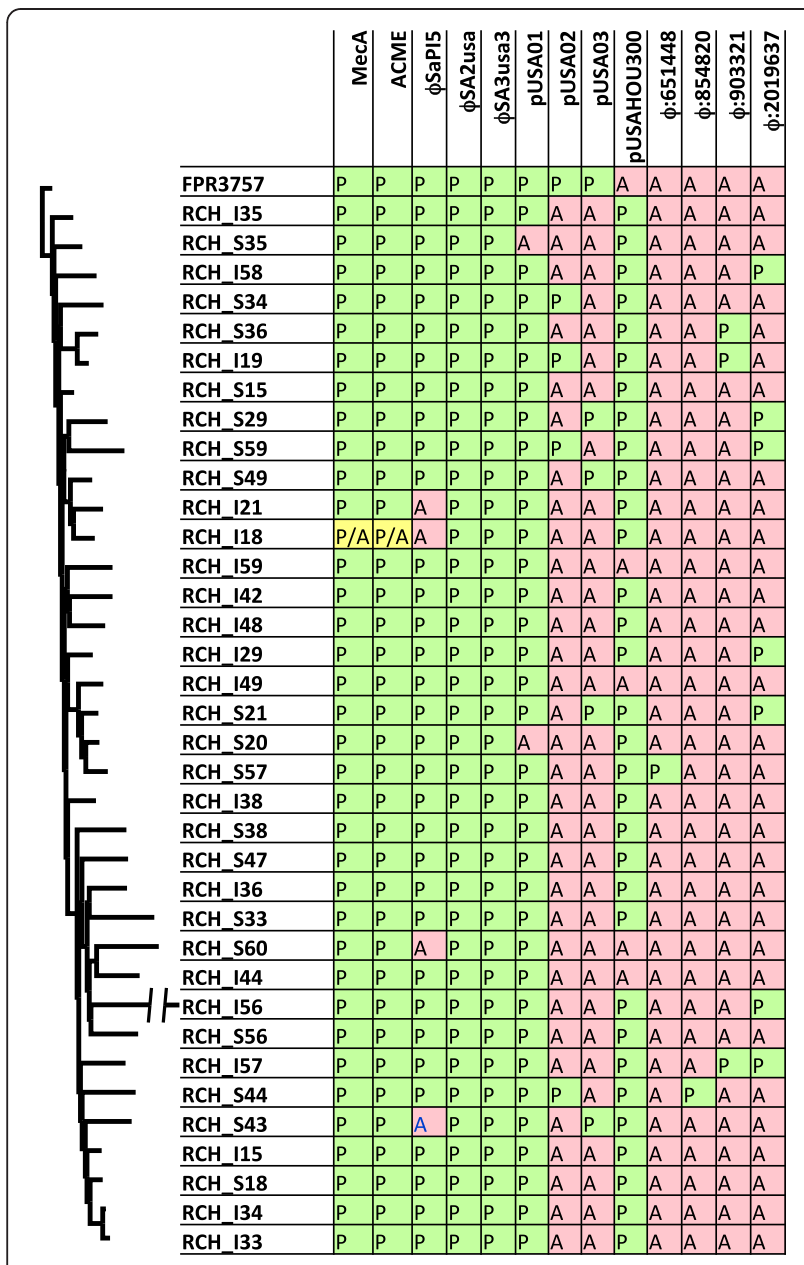

Figure 1 Table exhibiting homoplasy of large insertion/deletion events. The first 8 columns represent elements previously reported in USA300 isolates. The last 4 columns represent phage insertion sites discovered in our dataset (number denotes position in FPR3757). P and A represent presence or absence of the site respectively. The P/A for $\mathrm{RCH} I 18$ is a $21.6 \mathrm{~kb}$ deletion overlapping both the mec and ACME region but not entirely deleting either site.

clinical isolates. We verified the relocation using PCR and presume it to be an error in the original assembly of the FPR3757 reference sequence.

With respect to the clinical CA-MRSA isolates, the severe and SSTI groups each carried a mean of 71 and 80 single nucleotide variants $(\mathrm{SNV})$, respectively $(\mathrm{p}=0.03$, Analysis of Covariance with time as a covariate) (Table 1, Additional file 2: Figure S1). Of the 1054 SNV sites, $88 \%$ were private to a single isolate with $94 \%$ occurring at an allele frequency of less than 10\% (3 or fewer isolates). We performed multiple sequence alignment on all 36 isolates as well as the resequenced FPR3757 strain and constructed a maximum-likelihood phylogenetic tree (Figure 2). There is no obvious differentiation between severe and SSTI isolates with respect to their placements on the tree. However, there are two distinct haplogroups defined by 5 variants distributed across the genome. This suggests a decisive split in the lineage of the isolates from an ancestral clone. We screened our entire collection of severe $(n=36)$ and SSTI $(n=36)$ isolates for one of the eight haplogroup-defining variants and saw a modest association between the more recently arisen haplogroup $\mathrm{B}$ with the less severe (SSTI) isolates $(\mathrm{OR}=2.89,95 \%$ CI 1.2-7.0, $\mathrm{p}=0.03)$.

In both groups we observed a distinct temporal correlation supporting that the isolates are evolving away from a single common clone over time. We estimated an average mutation rate of $1.7 \times 10^{-6}$ ( $95 \%$ confidence interval, $4.5 \times 10^{-7}$ to $2.9 \times 10^{-6}$ ) per site per year for severe isolates and $2.6 \times 10^{-6}$ (95\% confidence interval, $9.1 \times 10^{-7}$ to $4.3 \times 10^{-6}$ ) per site per year for SSTI isolates (Figure 3). These values are in close concordance with a previously reported estimate of $3.3 \times 10^{-6}$ per site per year in hospitalacquired S. aureus [16]. Among SNVs, there was a transition: transversion ratio of $1.6: 1$ and a higher frequency of [ACGT]- > [AT] mutations, with a 1.9 fold greater occurrence, suggesting that CA-MRSA is actively introducing additional AT bias in an already GC depleted genome (Additional file 1: Table S4).

Small deletions ( $<100 \mathrm{bp})$ were 2 -fold more frequent than small insertions ( $<100 \mathrm{bp})$ among the 36 clinical isolates. As expected, we found that indels were most likely to occur in homopolymer stretches, consistent with polymerase slippage during replication [17]. Large deletions were detected in ten of the isolates. The largest deletion (21.6 kb), observed in a severe isolate, included a large portion of both the SCCmec and ACME regions but retained $p b p 2$ (the gene responsible for methicillin resistance). The ACME region has previously been postulated to be one of the loci responsible for USA 300's increased pathogenicity. While this locus may play a role in pathogenicity, our data suggest that the entire ACME region may not be required for severe infection. There was also a common $14 \mathrm{~kb}$ deletion completely removing staphylococcal pathogenicity island SaPI5 observed in 2 severe and 2 SSTI isolates. The deletion appeared on three separate bootstrapped supported branches of the phylogenetic tree, suggesting either recombination or three independent deletion events.

Large insertions ( $>100 \mathrm{bp})$ and additional contigs accounted for $606 \mathrm{~kb}$ of novel sequence across 15 isolates (6 severe and 9 SSTI isolates). For 12 isolates, we placed 14 insertions onto the core genome consisting of $269 \mathrm{~kb}$ of the additional sequence. Of the 14 placed insertions, 11 are large mobile genetic elements (MGEs) of prophage origin. There were four unique insertion sites for the 11 MGEs observed: 2 private appearing in only one isolate sequenced, 1 site shared by two isolates, and 1 site found in seven isolates at position $2.01 \mathrm{Mb}$ on the reference sequence (Figure 1). 


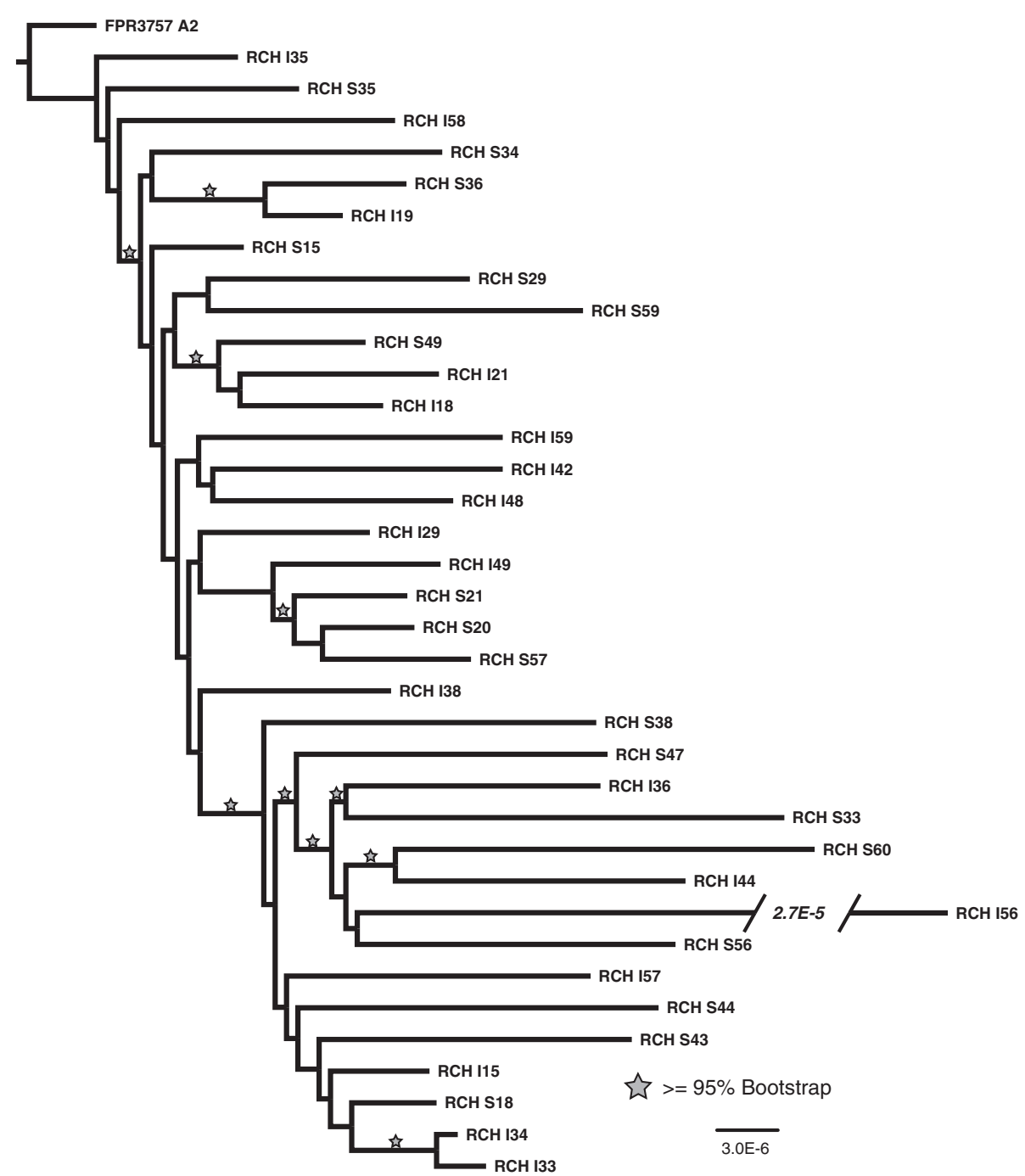

Figure 2 Maximum likelihood phylogenetic tree of the 36 clinical CA-MRSA isolates and the reference strain USA300-FPR3757. 100 bootstraps were performed and branches that carried 95\% support are starred. The tree was constructed from a 2872943 bp alignment with 126 informative sites. The severe isolate designated $\mathrm{RCH} \_156$ is truncated and the size of the truncation is listed on the tree.

Of the 7 isolates sharing the large insertion located at $2.01 \mathrm{Mb}$ the phylogenetic reconstruction suggests only two isolates share the insertion due to a single common ancestor with the remainder representing independent events. This insertion and the previously discussed $14 \mathrm{~kb}$ deletion represented two of the 11 events we detected as exhibiting homoplasy within our data (Additional file 1: Table S5). Unlike previous observations, we did not observe any convergent events with obvious implications for drug resistance [16]. This is not surprising in light of the fact that prior studies characterized HA-MRSA isolates that are presumably under different selective pressures than our pediatric CA-MRSA isolates from previously well children. Of the $9 \mathrm{SNVs}$ exhibiting homoplasy, only three affected protein-coding sequence directly. However, 4 of the 9 SNVs fell within or directly adjacent to transcriptional regulators, or within putative regulatory binding sequence. This suggests that regulatory mechanisms may play an equally critical role in CA-MRSA pathogenesis as point mutations conferring functional protein-coding changes.

\section{Functional distribution of mutations}

To assess the distribution of functional differences in our collection we annotated all non-ubiquitous variants of $100 \mathrm{bp}$ or smaller into three distinct classes: non-synonymous coding, synonymous coding, noncoding (Additional file 1: Table S6). In line with our discovery of a greater overall abundance of variants in the SSTI isolates we saw a significantly larger number of both synonymous and non-coding variants in the SSTI isolates when compared to the severe isolates. For 

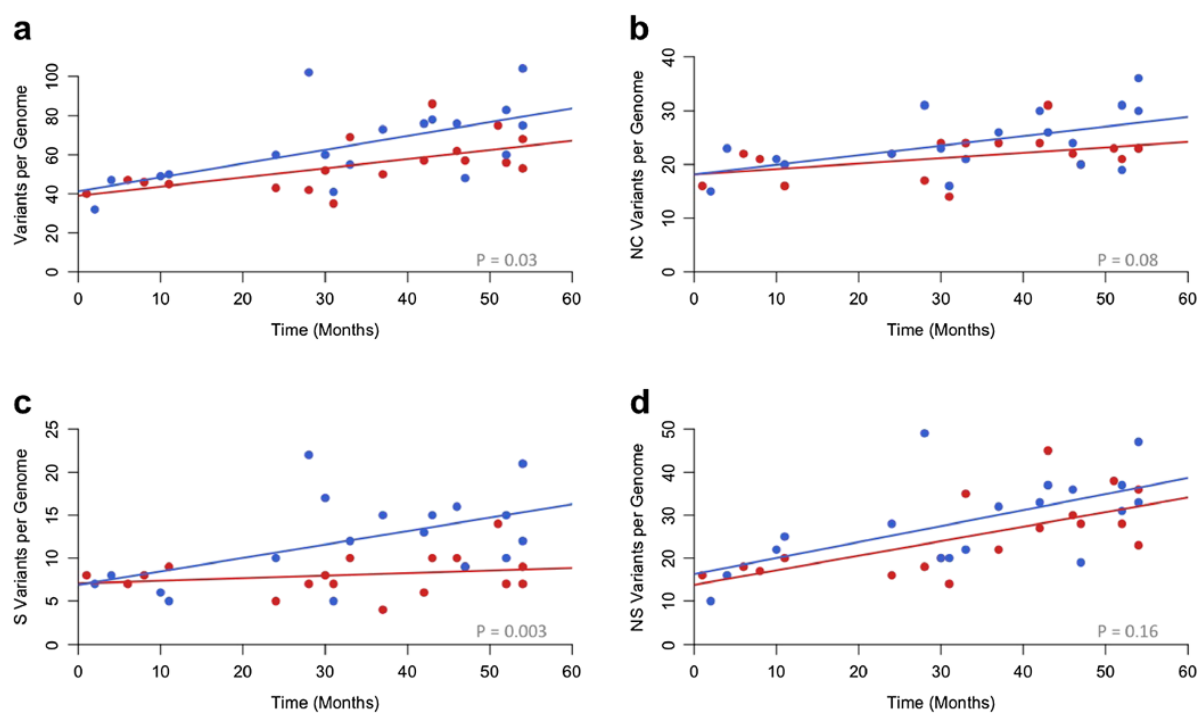

Figure 3 Assessment of both total and genic mutations of the 36 clinical CA-MRSA isolates. Number of total (a), noncoding (b) synonymous (c) and nonsynonymous (d) mutations in each of the isolates for the severe (red) and SSTI (blue) isolates are shown. Isolates are plotted according to their date of collection with the earliest isolate signifying month 1.

synonymous changes there were 219 variants in the SSTI isolates compared to 149 in the severe isolates $(\mathrm{p}=0.003$, Analysis of Covariance with time as a covariate). For non-coding changes we detected 434 changes in SSTI and 387 in the severe isolates $(p=0.08)$. For nonsynonymous variants the trend was similar with an over abundance of variants in the SSTI isolates $(\mathrm{SSTI}=522$, severe $=455)$ although this difference did not pass our threshold for significance $(p=0.16)$. The higher proportion of synonymous variants in the SSTI isolates relative to the difference in non-synonymous variants did create an overall lower $\mathrm{dN} / \mathrm{dS}$ ratio in our SSTI isolates (student's t-test, $\mathrm{p}=0.04$ ). However, we are cautious of any classical interpretation of the $\mathrm{dN} / \mathrm{dS}$ given the highly clonal nature of the samples and the small population size; both of which have been shown to cause fluctuation in the $\mathrm{dN} / \mathrm{dS}$ calculations $[18,19]$.

\section{Pathway analysis}

Under the hypothesis that the SSTI isolates are evolving away from a virulent phenotype we looked for an enrichment of genes harboring unique nonsense, ns-cSNVs or deleterious frame shift mutations among the SSTI isolates in genes related to virulence. Since no single gene harbored enough variants across sufficient number of isolates for direct testing, we sought to determine if there was enrichment across all virulence-associated genes classified as being involved in "pathogenesis" or "toxin production and resistance" by the JCVI CMR database [20]. This list consisted of 94 genes encompassing $4.9 \%$ of the total coding genome. We tested against only private variation among the isolates to prevent overt bias from the few mutations that are shared. There were 335 putative functional variants found in the SSTI isolates with 27 in genes related to virulence (Table 2). This represents a 1.66 fold increase over the expected value if the placement of variation was random across the genome (binomial test $\mathrm{p}<0.01$ ). In contrast the severe isolates carried close to the expected number at 13 variants out of 281, although we cannot claim a difference between the two groups given the number of isolates sampled (Chi squared of Invasive vs SSTI: $\mathrm{p}=0.1$ ).

\section{Discussion}

To address a major public health crisis that involves an infectious disease, it is essential that we track and better understand the biology of the causative pathogen. High-throughput DNA sequencing technologies provide a platform for tracking pathogens as they propagate and permit insights into virulence determinants which could be targeted pharmacologically. We sequenced the complete genomes of 36 emerging CA-MRSA isolates, half of which caused lethal or severe infections. As expected, we found evidence that all isolates of CA-MRSA are accumulating genetic variation over time, indicative of an evolution away from an ancestral clone. This rate corresponded to what has been seen in a similar study for HA-MRSA suggesting that, at least at a population wide level, these two distinct classes are evolving at a similar rate [16]. However at higher resolution these two groups likely have distinct pressures being applied to them given their differing environments. One such example is the lack of homoplasy within genes related to antimicrobial resistance. In addition we did not 
Table 2 Variants in virulence related genes

\begin{tabular}{|c|c|c|c|c|c|c|c|}
\hline Gene & Gene ID & Position & Ref base & Alt base & Substitution & Invasive & SSTI \\
\hline immunoglobulin G binding protein A precursor & SAUSA300_0113 & 128554 & G & $\mathrm{T}$ & N380K & 0 & 1 \\
\hline lucC family siderophore biosynthesis protein & SAUSA300_0123 & 142320 & G & $\mathrm{T}$ & S4041 & 1 & 0 \\
\hline sucrose-specific PTS tranporter protein & SAUSA300_0194 & 226384 & C & $\mathrm{T}$ & A153V & 1 & 0 \\
\hline sucrose-specific PTS tranporter protein & SAUSA300_0194 & 226642 & G & A & G239D & 0 & 1 \\
\hline choloylglycine hydrolase family protein & SAUSA300_0269 & 321604 & C & A & P194T & 0 & 1 \\
\hline essC protein & SAUSA300_0283 & 336391 & G & A & E142K & 0 & 1 \\
\hline essC protein & SAUSA300_0283 & 336694 & A & G & $1243 \mathrm{~V}$ & 1 & $\overline{0}$ \\
\hline superantigen-like protein 5 & SAUSA300_0399 & 451121 & C & A & A7E & 1 & 1 \\
\hline superantigen-like protein & SAUSA300_0407 & 460962 & TACAG & -—- & Frameshift 108 & 11 & 10 \\
\hline dimethyladenosine transferase & SAUSA300_0470 & 530052 & $\mathrm{~T}$ & A & V258E & 0 & 1 \\
\hline clumping factor $\mathrm{A}$ & SAUSA300_0772 & 859497 & C & A & A117D & 0 & 1 \\
\hline enterotoxin Q & SAUSA300_0801 & 884359 & $\mathrm{~T}$ & - & Frameshift 142 & 1 & $\overline{0}$ \\
\hline cysteine protease precursor & SAUSA300_0950 & 1038844 & A & $\mathrm{T}$ & V206E & 0 & 1 \\
\hline fmt protein & SAUSA300_0959 & 1051797 & C & $\mathrm{T}$ & T269| & 0 & 1 \\
\hline alpha-hemolysin precursor & SAUSA300_1058 & 1157169 & G & $\mathrm{T}$ & $\mathrm{T} 53 \mathrm{~N}$ & 0 & $\overline{1}$ \\
\hline putative fibronectin/fibrinogen binding protein & SAUSA300_1101 & 1204860 & C & A & D555Y & 0 & 2 \\
\hline putative enterotoxin type $A$ & SAUSA300_1559 & 1708640 & C & A & E78* & 1 & 0 \\
\hline cell wall surface anchor family protein & SAUSA300_1702 & 1878567 & G & A & P1968S & 1 & 0 \\
\hline cell wall surface anchor family protein & SAUSA300_1702 & 1878783 & G & C & Q1896E & 0 & 1 \\
\hline cell wall surface anchor family protein & SAUSA300_1702 & 1878930 & C & A & A1847S & 0 & 1 \\
\hline cell wall surface anchor family protein & SAUSA300_1702 & 1880436 & C & $\mathrm{T}$ & E1345K & 0 & 1 \\
\hline cell wall surface anchor family protein & SAUSA300_1702 & 1880783 & G & A & A1229V & 0 & 1 \\
\hline serine protease SplA & SAUSA300_1758 & 1943333 & $\mathrm{~T}$ & C & D113G & 0 & 1 \\
\hline lantibiotic epidermin biosynthesis protein EpiC & SAUSA300_1765 & 1950378 & A & G & V209A & 0 & 1 \\
\hline lantibiotic epidermin biosynthesis protein EpiB & SAUSA300_1766 & 1953206 & $\mathrm{~T}$ & - & Frameshift 261 & 0 & 1 \\
\hline leukotoxin LukD & SAUSA300_1768 & 1955543 & C & $\mathrm{T}$ & V254I & 1 & 0 \\
\hline leukotoxin LukE & SAUSA300_1769 & 1957230 & $\mathrm{~T}$ & C & K4E & 0 & 1 \\
\hline Aerolysin/leukocidin family protein & SAUSA300_1975 & 2130674 & G & A & A63V & 1 & 0 \\
\hline Aerolysin/leukocidin family protein & SAUSA300_1975 & 2130847 & $\mathrm{~T}$ & - & Frameshift 5 & 0 & 1 \\
\hline hyaluronate lyase precursor & SAUSA300_2161 & 2338286 & G & $\mathrm{T}$ & K65N & 0 & 1 \\
\hline hyaluronate lyase precursor & SAUSA300_2161 & 2340008 & A & $\mathrm{T}$ & L639F & 1 & 0 \\
\hline AcrB/AcrD/AcrF family protein & SAUSA300_2213 & 2380381 & - & $\mathrm{T}$ & Frameshift 1050 & 0 & 1 \\
\hline AcrB/AcrD/AcrF family protein & SAUSA300_2213 & 2382879 & A & $\mathrm{T}$ & L218* & 0 & 1 \\
\hline $\begin{array}{l}\text { teicoplanin resistance associated membrane } \\
\text { protein TcaB protein }\end{array}$ & SAUSA300_2301 & 2473522 & C & G & W311C & 0 & 1 \\
\hline IgG-binding protein SBI & SAUSA300_2364 & 2540728 & G & A & E150K & 0 & 1 \\
\hline IgG-binding protein SBI & SAUSA300_2364 & 2540773 & C & A & Q165K & 1 & 0 \\
\hline IgG-binding protein SBI & SAUSA300_2364 & 2540911 & G & A & V211M & 1 & $\overline{1}$ \\
\hline gamma-hemolysin component $\mathrm{A}$ & SAUSA300_2365 & 2542364 & C & A & Q80K & 1 & 0 \\
\hline gamma-hemolysin component $\mathrm{C}$ & SAUSA300_2366 & 2543638 & A & G & $16 \mathrm{~V}$ & 0 & 1 \\
\hline putative transporter & SAUSA300_2406 & 2591556 & A & C & M273R & 2 & 0 \\
\hline clumping factor $B$ & SAUSA300_2565 & 2774393 & C & $\mathrm{T}$ & G883R & 0 & 1 \\
\hline
\end{tabular}

observe differences between the $\mathrm{dN} / \mathrm{dS}$ ratios of the core and non-core portions of the genome as previously reported [21]. However, given the different population of samples and the smaller sample set that our data represents, neither discordance is troubling. We did detect that the accumulated genetic variation appears to be higher among CA-MRSA isolates causing the milder SSTI phenotypes. This distance also manifested itself by 
association of a distinct haplogroup, which evolved from the parental strain, with the less severe sample. Further, pathways involved in virulence are enriched among genes harboring coding variations among the clinical isolates of lesser clinical severity. Taken together, these data suggest that the invasiveness of USA 300 may be dependent, to some degree, on the genetic distance a particular clone is away from the clonal parental USA 300 strain. USA 300 was first described a little over a decade ago suggesting its emergence is recent [22,23]. The strain is a result of a two step process; first through the acquisition of the SCCmex IV complex which resulted in the clonal type USA500, followed later by the acquisition of several genes including the PVL and ACME loci [24]. Given that Staphylococcus aureus is a natural member of our nasal flora, as demonstrated by pervasive asymptomatic carriage $[25,26]$, there is likely genetic pressure on the organism to maintain some degree of a commensal relationship and as a result dampen the hyper-virulent characteristic which arose from a single recombination event. This "wave" of clonality wherein an epidemic clone decreases it pathogenicity and as a result its association with infections independent of control measures has been seen in other MRSA strains [27]. Thus, the increase in genetic diversity of USA300 SSTI isolates may be an evolutionary progression away from being the hypervirulent clonal type that USA300 represents.

The fact that we did not find any single genetic variant or set of genetic variants readily capable of distinguishing severe- and SSTI associated CA-MRSA isolates speaks to the complexity of MRSA pathogenicity and underscores the need for more sophisticated ways to identify disease-causing CA-MRSA strains via genomic screens. While we focused our analysis to only a small number of CA-MRSA isolates displaying distinct clinical phenotypes, we are optimistic that surveying a large number of isolates (including CA-MRSA asymptomatic carrier- and MSSA-assocaited isolates) in the future will provide the power to detect evolutionary pressures at the single gene level. Of course there are two genomes at play during an infection and, as a result, a greater understanding of human genetic variation is also pivotal for the complete picture of how genetic architecture influences our interaction with microbial pathogens. It is also important to recognize that numerous non-genetic influences for virulence exist; as such to effectively investigate the genetics of pathogenicity it is critical to properly select the cases as to mitigate such effects of predisposition.

\section{Conclusions}

Technological advances and continued decreases in the cost of sequencing are allowing the whole genome reconstruction of large collections of pathogens. These data sets offer valuable insight into the clonal population structure as well clues into the evolutionary pressures applied to the pathogen and will provide a foundation for the use of whole genome sequencing in a clinical setting. In the future, the genomic identity of individual isolates may be increasingly compared to global pathogen databases allowing the rapid identification of strains likely to cause severe disease, possess drug-resistance, and additional traits of critical utility to inform clinical decisions.

\section{Methods}

\section{Isolate selection}

For the five year period from January 1, 2003 to December 31, 2007, we identified two sets of children infected by CA-MRSA: (1) those with severe infections and (2) those with SSTI. Severe infections were defined as those causing invasive, potentially life-threatening clinical disease, including: complicated pneumonia (necrosis, empyema, or lung abscess); endocarditis (defined as persistent bacteremia with echocardiographic findings); deep tissue invasive abscess (mediastial, perinephric); osteoarticular infection; and pyomyositis (Additional file 1: Table S2). SSTI were defined as soft-tissue abscesses not associated with severe disease (as above) but requiring incision and drainage.To populate these two study groups, sterile-site (blood, bone, joint fluid, tracheal aspirate, pleural fluid, invasive abscess, soft-tissue abscess) MRSA isolates obtained from individual, healthy children aged 0-18 years were included. Non-sterile site (e.g. nasopharynx, stool) and polymicrobial infections were excluded. Isolates obtained from children with chronic diseases or immunocompromised states (diabetes, cystic fibrosis, neoplasm, etc.), from vascular catheters or catheter-sites and nosocomial isolates (onset after hospital admission) were also excluded.

\section{Demographic and clinical data}

Clinical and laboratory data for all patients were collected on a standardized data collection form including: age; gender; self-identified ethnicity; infection site; length of stay (including pediatric intensive care unit stay); inpatient and discharge antibiotic treatment regimen and duration; outcome; and long-term sequelae (Additional file 1: Table S1).

\section{Specimen processing}

Frozen MRSA specimens were thawed and inoculated onto blood-agar plates with two rounds of subculturing to ensure optimal growth. Colonies were then transferred into enriched brain/heart infusion broth media. DNA extraction was performed using a with DNeasy blood and tissue kits with lysostaphin replacing lysozyme during the initial lysis. (Qiagen, Germantown, MD). 


\section{Illumina sequencing and preparation}

For each of the 36 samples Illumina library preperation started with 5 ug of extracted DNA quantified by 260/ 280 (nanodrop). The standard Illumina protocol was followed through using reagents from New England Biosciences with the following exceptions: samples were sheared by either nebulization or adaptive focused acoustics (Covaris), sequencing adaptors added after a-tailing contained an addition 4 bp nucleotide barcode on the 3 ' end allowing for the multiplexing of up to 4 samples per lane. After library preparation paired-end sequencing was performed for 40 cycles on each read and images were processed using Illumina pipeline 1.4. Data for each isolate can be accessed at http://sites.google.com/site/ ryantewhey/home/data.

\section{Sequence assembly}

Genome assembly utilized a mapping/denovo hybrid approach consisting of the following; Reads were first mapped using BWA [13] and default parameters to the reference FPR3757 core genome its three plasmids and the pHOU300 plasmid. Plasmids with little or no coverage were discarded. Variants and small indels with a BWA consensus score of 30 or greater and a mapping score of 10 or greater were incorporated into the sequence. We also utilized custom variant calling pipelines for further refinement of insertion/deletion detection. Mapping quality was then assessed across the genome and detected plasmids. The genome was then broken into 'mapping contigs' where paired-end information was discordance (outside the expected size distribution), mapping quality was high and spanning coverage was low. Separately reads were processed using the Abyss [14] assembly package using default parameters and a kmer of 23. Only assembled contigs of 1000 bp or greater were used for subsequent analysis.

Using a combination of BLAT [28] and the AMOS assembly package (http://amos.sourceforge.net), mapping and de novo contigs were merged to produce a consensus contig. In instances were the mapping and denovo contigs differed two separate contigs were produced. Reads were then mapped over the two assemblies (mapping consensus \& denovo consensus) separately with BWA allowing only perfect matches. The location was then assessed at both variants for mapping quality and the variant with the greatest support via mapping was chosen.

Contigs were then scaffolded using the FPR3757 reference genome and multiple alignment was performed using the progressive Mauve engine of Mauve [29] followed by visual inspection and realignment.

\section{Sequence analysis}

Prior to creating a phylogenetic tree all insertions and deletions were removed from the sequence and recoded as a single nucleotide transversion polyphorphism at the site of the event. A maximum likelihood tree was then constructed using RAxML [30] with 100 bootstraps on the CIPRES portal (http://www.phylo.org/). The tree was visualized and rooted using FigTree software (http://tree. bio.ed.ac.uk/software/figtree/).

For all variant analysis 24 variants were seen in all 36 clinical isolates and were removed from all subsequent analysis. In addition, 84 single nucleotide variants and 1 insertion were found clustered together within a $10 \mathrm{~kb}$ window of isolate I56. These variants most likely represent a single event such as recombination and not multiple unique events. Because of this they were removed from all variant analysis.

For the calculation of the $\mathrm{dN} / \mathrm{dS}$ ratio we used the formula $(\mathrm{N} / \mathrm{n}) /(\mathrm{S} / \mathrm{s})$, where $\mathrm{N}$ and $\mathrm{S}$ are the number of nonsynonymous and synonymous mutations respectively found in the sequenced isolates, $\mathrm{n}$ is the total possible nonsynonymous mutations and $\mathrm{s}$ is the total number of possible synonymous mutations in the FPR3757 core genome. Significance of selection between groups of isolates was calculated with a two-tailed t-test. Mutation rate differences between the two isolate groups were calculated from the interaction effects of a standard linear regression. Correlation within strains to synonymous, nonsynonymous and noncoding mutations were calculated from Spearman's non-parametric correlation coefficient.

Primary and secondary gene role categories were assigned to genes based on the J. Craig Venter Institute Comprehensive Microbial Resource annotation of Staphylococcus aureus USA300-FPR3757. Enrichment probabilities were calculated based on the binomial distribution, where the observed number nonsynonymous, nonsense, and frameshift mutations within a pathway was compared to the expectation due to random chance. The expected proportion of mutations occurring within a pathway is defined as the sum of the lengths of the genes belonging to the pathway over the sum of the length of all genes within the Staphylococcus aureus USA300-FPR3757 genome. Regions of the genome masked out during the sequence assembly and variant calling phases were not included in the pathway or whole genome summations.

\section{Haplotype genotyping}

To genotype the larger collections of 36 severe and 36 SSTI isolates we tested one of the eight variants that differentiated between the two haplogroups. Standard PCR was performed for 35 cycles with Phusion mastermix (Finnzymes) and $100 \mathrm{nM}$ of Primer A (GCAGCAATAC CACCGAAAAT) and Primer B (GCGCAAGCTAGTGG GATAAG). PCR products were purified with Agencourt AMPure beads (Beckman Coulter Genomics) and 
subsequently digested with SpeI (New England Biolabs) overnight at $37 \mathrm{C}$. The genotype was then visually scored on a agarose gel with the uncut product corresponding to haplotype A of the reference strain FPR3757.

\section{Additional files}

Additional file 1: Table S1. Patient characteristics of sequence isolates by study group. Table S2: Clinical diagnoses of included CA-MRSA severe infections. Table S3: Site of included CA-MRSA severe infections. Table S4: Nucleotide mutation catagories. Table S5: Variants exhibiting convergent evolution. Table S6: Protein Altering Single Nucleotide Variants.

Additional file 2: Figure S1. Pangenomic representation of all 36 clinical isolates relative to the USA300 FPR3757 reference.

\section{Competing interests}

The authors declare that they have no competing interests.

\section{Authors' contributions}

RT, CRC, JADL, EJT, JSB and NJS designed and supported the project. CRC identified and reviewed all clinical isolates. RT and CRC performed laboratory work. RT, VB and AT performed data analysis. RT and CRC wrote the manuscript with additions from all other authors. All authors read and approved the final manuscript.

\section{Acknowledgements}

The authors would like to thank M. Miller at San Diego Supercomputer Center for computational support. This work was funded in part by the Scripps Translational Sciences Institute Clinical Translational Science Award [grant number UL1 RR025774-03] and Scripps Genomic Medicine.

\section{Author details}

'Scripps Genomic Medicine, Scripps Translational Science Institute, La Jolla, CA, USA. ${ }^{2}$ Department of Molecular and Experimental Medicine, The Scripps Research Institute, La Jolla, CA, USA. ${ }^{3}$ Division of Biological Sciences, University of California, San Diego, La Jolla, CA, USA. ${ }^{4}$ Department of Pediatrics, Division of Infectious Diseases, Rady Children's Hospital San Diego, San Diego, CA, USA. ${ }^{5}$ Department of Pediatrics, Division of Infectious Diseases, University of California, San Diego, CA, USA. ${ }^{6}$ Department of Pediatrics, Division of Hospital Medicine, Rady Children's Hospital San Diego, San Diego, CA, USA.

Received: 3 May 2012 Accepted: 14 September 2012

Published: 25 September 2012

\section{References}

1. Chambers HF, Deleo FR: Waves of resistance: staphylococcus aureus in the antibiotic era. Nat Rev Microbiol 2009, 7(9):629-641.

2. Klein E, Smith DL, Laxminarayan R: Hospitalizations and deaths caused by methicillin-resistant staphylococcus aureus, United States, 1999-2005. Emerg Infect Dis 2007, 13(12):1840-1846.

3. Klevens RM, Morrison MA, Nadle J, Petit S, Gershman K, Ray S, Harrison LH, Lynfield R, Dumyati G, Townes JM, et al: Invasive methicillin-resistant staphylococcus aureus infections in the United states. JAMA 2007, 298(15):1763-1771.

4. Moran GJ, Krishnadasan A, Gorwitz RJ, Fosheim GE, McDougal LK, Carey RB, Talan DA: Methicillin-resistant $\mathrm{S}$. aureus infections among patients in the emergency department. N Engl I Med 2006, 355(7):666-674.

5. Lee SM, Ender M, Adhikari R, Smith JM, Berger-Bachi B, Cook GM: Fitness cost of staphylococcal cassette chromosome mec in methicillin-resistant Staphylococcus aureus by way of continuous culture. Antimicrob Agents Chemother 2007, 51(4):1497-1499.

6. Collins J, Rudkin J, Recker M, Pozzi C, O'Gara JP, Massey RC: Offsetting virulence and antibiotic resistance costs by MRSA. ISME J 2010, 4(4):577-584.

7. Diep BA, Gill SR, Chang RF, Phan TH, Chen JH, Davidson MG, Lin F, Lin J, Carleton HA, Mongodin EF, et al: Complete genome sequence of USA300, an epidemic clone of community-acquired meticillin-resistant staphylococcus aureus. Lancet 2006, 367(9512):731-739.

8. Otter JA, French GL: Molecular epidemiology of community-associated meticillin-resistant staphylococcus aureus in Europe. Lancet Infect Dis 2010, 10(4):227-239.

9. Larsen A, Stegger M, Goering R, Sorum M, Skov R: Emergence and dissemination of the methicillin resistant staphylococcus aureus USA300 clone in Denmark (2000-2005). Euro Surveill 2007, 12(2):22-24

10. Hota B, Lyles R, Rim J, Popovich KJ, Rice T, Aroutcheva A, Weinstein RA: Predictors of clinical virulence in community-onset methicillin-resistant staphylococcus aureus infections: the importance of USA300 and pneumonia. Clinical infectious diseases: an official publication of the Infectious Diseases Society of America 2011, 53(8):757-765.

11. Kennedy AD, Otto M, Braughton KR, Whitney AR, Chen L, Mathema B, Mediavilla JR, Byrne KA, Parkins LD, Tenover FC, et al: Epidemic community-associated methicillin-resistant staphylococcus aureus: recent clonal expansion and diversification. Proc Natl Acad Sci USA 2008, 105(4):1327-1332.

12. Hall TA, Sampath R, Blyn LB, Ranken R, Ivy C, Melton R, Matthews H, White $\mathrm{N}$, Li F, Harpin V, et al: Rapid molecular genotyping and clonal complex assignment of staphylococcus aureus isolates by PCR coupled to electrospray ionization-mass spectrometry. J Clin Microbiol 2009, 47(6):1733-1741.

13. Li H, Durbin R: Fast and accurate short read alignment with Burrows-Wheeler transform. Bioinformatics 2009, 25(14):1754-1760.

14. Simpson JT, Wong K, Jackman SD, Schein JE, Jones SJ, Birol I: ABySS: a parallel assembler for short read sequence data. Genome Res 2009, 19(6):1117-1123.

15. Highlander SK, Hulten KG, Qin X, Jiang H, Yerrapragada S, Mason EO Jr, Shang Y, Williams TM, Fortunov RM, Liu Y, et al: Subtle genetic changes enhance virulence of methicillin resistant and sensitive staphylococcus aureus. BMC Microbiol 2007, 7:99.

16. Harris SR, Feil EJ, Holden MT, Quail MA, Nickerson EK, Chantratita N, Gardete S, Tavares A, Day N, Lindsay JA, et al: Evolution of MRSA during hospital transmission and intercontinental spread. Science 2010, 327(5964):469-474.

17. Viguera E, Canceill D, Ehrlich SD: Replication slippage involves DNA polymerase pausing and dissociation. EMBO J 2001, 20(10):2587-2595.

18. Kryazhimskiy S, Plotkin JB: The population genetics of dN/dS. PLoS Genet 2008, 4(12):e1000304

19. Kuo $\mathrm{CH}$, Moran NA, Ochman $\mathrm{H}$ : The consequences of genetic drift for bacterial genome complexity. Genome Res 2009, 19(8):1450-1454.

20. Davidsen T, Beck E, Ganapathy A, Montgomery R, Zafar N, Yang Q, Madupu R, Goetz P, Galinsky K, White O, et al: The comprehensive microbial resource. Nucleic Acids Res 2010, 38(Database issue):D340-345.

21. Castillo-Ramirez S, Harris SR, Holden MT, He M, Parkhill J, Bentley SD, Feil EJ: The impact of recombination on $\mathrm{dN} / \mathrm{dS}$ within recently emerged bacterial clones. PLoS Pathog 2011, 7(7):e1002129.

22. CDC: Methicillin-resistant staphylococcus aureus infections among competitive sports participants-Colorado, Indiana, Pennsylvania, and Los Angeles County, 2000-2003. MMWR Morb Mortal Wkly Rep 2003, 52(33)::793-795.

23. CDC: Methicillin-resistant staphylococcus aureus skin or soft tissue infections in a state prison-Mississippi, 2000. MMWR Morb Mortal Wkly Rep 2001, 50(42):919-922.

24. Li M, Diep BA, Villaruz AE, Braughton KR, Jiang X, DeLeo FR, Chambers HF, Lu Y, Otto M: Evolution of virulence in epidemic community-associated methicillin-resistant staphylococcus aureus. Proc Natl Acad Sci USA 2009, 106(14):5883-5888

25. Hussain FM, Boyle-Vavra S, Daum RS: Community-acquired methicillin-resistant staphylococcus aureus colonization in healthy children attending an outpatient pediatric clinic. Pediatr Infect Dis J 2001, 20(8):763-767.

26. Kenner J, O'Connor T, Piantanida N, Fishbain J, Eberly B, Viscount H, Uyehara C, Hospenthal D: Rates of carriage of methicillin-resistant and methicillin-susceptible staphylococcus aureus in an outpatient population. Infect Control Hosp Epidemiol 2003, 24(6):439-444.

27. Wyllie D, Paul J, Crook D: Waves of trouble: MRSA strain dynamics and assessment of the impact of infection control. J Antimicrob Chemother 2011, 66(12):2685-2688. 
28. Kent WJ: BLAT-the BLAST-like alignment tool. Genome Res 2002 12(4):656-664

29. Darling AC, Mau B, Blattner FR, Perna NT: Mauve: multiple alignment of conserved genomic sequence with rearrangements. Genome Res 2004 14(7):1394-1403

30. Stamatakis A: RAxML-VI-HPC: maximum likelihood-based phylogenetic analyses with thousands of taxa and mixed models. Bioinformatics 2006, 22(21):2688-2690.

doi:10.1186/1471-2164-13-508

Cite this article as: Tewhey et al:: Genetic structure of community

acquired methicillin-resistant Staphylococcus aureus USA300. BMC Genomics 2012 13:508.

\section{Submit your next manuscript to BioMed Central and take full advantage of:}

- Convenient online submission

- Thorough peer review

- No space constraints or color figure charges

- Immediate publication on acceptance

- Inclusion in PubMed, CAS, Scopus and Google Scholar

- Research which is freely available for redistribution 